\title{
THE EFFECTS OF SALT ON BREAD: TECHNOLOGICAL CONSIDERATIONS FOR REDUCED SALT LEVELS
}

\author{
Helen Tuhumury \\ Program Studi Teknologi Hasil Pertanian Fakultas Pertanian-Universitas Pattimura \\ helentuhumuri@yahoo.com
}

\begin{abstract}
Salt is a dietary mineral composed primarily of sodium chloride. These two components of salt are essential nutrient for humans with important functions in regulating extracellular fluid volume and the active transport of molecules across cell membranes. However, having too much salt in the diet increases the risk of health problems. High sodium intake is positively correlated with the level of blood pressure. Therefore, it is necessary to consume food with reduced salt content. Salt has been used as a food preservative that kills or limits of foodborne pathogens and spoilage organism by decreasing water activity. Salt also has functions in foods by adding flavor and masking bitter tastes, controlling growth of yeast and strengthening the dough network and gas retention of the dough. Reducing salt content in processed food especially for bread based products is one of the greatest ongoing challenges facing food manufactures. It also has been difficult to reduce or remove salt or sodium chloride from bread since salt is one of the four essential ingredients in bread. Reducing salt level from $1.2 \%$ does not significantly affect dough rheological properties and breadmaking quality. However, omission of salt entirely leads to significant reduction in dough, bread quality and its palatability.
\end{abstract}

Keywords: effect, salt, bread.

\section{INTRODUCTION}

This review investigates the importance of salt inclusion in cereal products particularly bread and the difficulties associated with reducing salt in the products. The report focuses on the effect of salt on dough rheological properties, baking properties and taste of the bread.

Salt is a dietary mineral composed primarily of sodium chloride. These two components of salt are essential for humans, however having too much salt in the diet increases the risk of health problems. High sodium intake is positively correlated with the level of blood pressure (Elliott, et al., 1996). In addition there are well established relationships of usual blood pressure levels with the risks of vascular problems (MacMahon, et al., 1990; Tuomilehto, et al., 2001). There is convincing evidence that reduced salt intake significantly benefits health. Trials have demonstrated the beneficial effects of reduced dietary sodium intake 
Helen: The effects of salt on bread

on blood pressure when low salt diets are adhered to in the long term (Law, Frost, \& Wald, 1991). In addition, the World Health Organization (WHO) has made specific recommendations regarding the implementation of national salt reduction programs (WHO, 2007). Therefore, it is necessary to consume food with reduced salt content. Despite the risks of high dietary salt intake, salt is often consumed above the levels required for good health (6 g/day) by consumers in Australia and other developed nations (Webster, et al., 2009). It has been estimated that around $75 \%$ of the salt we eat is already in the foods we buy which are mostly contained in processed food (Dotsch, et al., 2009; James, Ralph, \& SanchezCastillo, 1987). In fact, $25 \%$ salt intake in the western diet are from cereal products (Miller \& Hoseney, 2008). It is important then for food manufacturers to develop products with reduced salt content.

However, sodium reduction is not that easy since salt not only has an important role in taste of the food but also for preservation, and in some products structuring, or other purposes (Dotsch, et al., 2009). Reductions in the sodium content of processed foods has been difficult to achieve because food manufacturers usually perceive the sodium content to be vital to the flavour and acceptability of the product (Breslin \& Beauchamp, 1997). In addition, the inclusion of salt in solid food and dry powder formulations generally has a significant technological role in the processing and stability of these products. Therefore, reducing salt content in processed food especially for cereal based products is one of the greatest ongoing challenges facing food manufacturers. It is not sufficient to simply reduce or remove salt from the formulation of a product and expect product characteristics such as appearance, taste, flavour and texture to be maintained.

\section{The effect of salts on dough \\ rheological properties}

Reducing or removing salt or sodium chloride from bread has been difficult, since salt is one of the four essential ingredients in bread (flour, salt, yeast and water) (Miller \& Hoseney, 2008). It is essential because salt affects the dough properties, baking or bread properties and its palatability. However, there are conflicting results about the relation of salt's effect on dough properties and baking properties. 
Turning first to effect of salt on the dough properties, salt is usually added to form dough with other ingredients before it is exposed to baking process. The dough properties are influenced by salt in ways that salt usually stabilize the yeast fermentation rate, strengthening the dough and increasing dough mixing time (Miller \& Hoseney, 2008). Those effects on dough are studied by looking at dough rheology when salt is added into the formulation for bread making. The studies mainly concentrate on the dough properties alone without any correlation to the baking properties.

Firstly, salt inhibit or controls fermentation rate by decreasing the rate of gas production which result in the longer proof times (He, Roach, \& Hoseney, 1992). This appears to be the result of increased osmotic pressure and the sodium and chloride ions on the membrane of yeast cells (Miller \& Hoseney, 2008). The growth of yeast cell is then retarded; hence the fermentation and dough development is controlled. If dough is made without salt, the yeast ferments excessively resulting in gassy and sour dough. The dough with these properties when baked may result in products with open grain and poor texture (Miller \& Hoseney, 2008) He et al., (1992) highlight the effect of dough with and without salt and different kinds of salt on the gas production. Indeed, dough without salt has higher gas production $67.8 \mathrm{GU}$ and was significantly different compared to dough treated with sodium chloride which had 61.8 GU. In addition, salt with different cations has lower gas production than sodium chloride except for potassium chloride. This study does not indicate whether it is acceptable to reduce salt from the formulation without posing any effect on gas production.

Another study using a Chopin Rheofermentometer was applied to obtain information on gas expansion and retention capabilities of dough during fermentation. The effect of different salt concentrations in reducing series on dough properties and yeast growth were measured simultaneously. (Lynch, Dal Bello, Sheehan, Cashman, \& Arendt, 2009). The results showed that the maximum height of dough that can be considered as a marker for baking quality increased as salt level decreased. As mentioned earlier that salt inhibit yeast growth, thus a reduction in salt levels increase yeast activity hence 
the higher $\mathrm{CO}_{2}$ production. To achieve good quality bread, it is necessary to control gas production to a minimum level. Therefore, reducing salt level may bring poor quality. However, even though reduced salt level result in higher gas production, the capability of a dough to retain gas was lower as shown by lower retention coefficient when salt content of the dough was reduced (Lynch, et al., 2009).

Secondly, salt usually strengthens dough. The effects of salt addition on dough are primarily attributed to the interaction of salt with gluten protein (Fu, Sapirstein, \& Bushuk, 1996; He, et al., 1992; Larsson, 2002). When dough is prepared without salt, the gluten protein has positive net charges in the flour-water system. These positive charges repulse each and keep the protein molecule to interact which may result in weaker dough network. When salt is present, it shields the charges on the gluten protein, reducing electrostatic repulsion between proteins and allowing them to associate thus producing stronger dough (Kinsella \& Hale, 1984; Miller \& Hoseney, 2008). The studies show that salt is important to maintain stronger dough. However, still they do not present the effect of reduced salt content on the dough properties.

The study by Lynch et al. (2009) showed that reducing level of salt do not significantly affect the resistance to extension and extensibility of dough, however there is a tendency that reduced salt level result in lower resistance to extension and extensibility as a result of weaker gluten network. The rheology of the dough added with reduced salt levels was also studied and showed that the elastic modulus $\left(G^{\prime}\right)$ increased with decreasing salt addition. However, Larsson (2002) showed that there is a moderate but significant decrease $G^{\prime}$ value with reduced salt levels. The contrasting results of these studies might be due to the different quality of flour used. In fact, the effect of salt on dough also determined by the quantity and the quality of the protein used (Wu, Beta, \& Clarke, 2006). Since these two studies using the same amount of flour, then the differences are more readily associated with the different quality of flour used. Overall, it can be said that no major structural changes in the dough take place as a result of salt reduction, despite the quality of flour used. Therefore, the reduced salt content is possible to be 
Helen: The effects of salt on bread

used in the formulation of bread depending on the type of the flour used whether it is high protein flour or low one.

Thirdly, salt affect dough properties in terms of mixing requirements. Several studies showed that salt increases the mixing time of the dough as shown by farinograph results (Preston, 1989; Salovaara, 1982). Dough prepared without salt makes gluten proteins hydrate faster since they repulse each other. The dough then is more readily mixed in just a short time. But, when salt is added to the formulation, the gluten proteins associate each other and make them hydrate more slowly resulting in longer mixing time (Miller \& Hoseney, 2008). There is no specific study that shows the effect reduced salt levels on the dough mixing time. However, it can be said that higher salt content may result in longer mixing time. The longer mixing time can pose a problem to bread manufacturers who run large bakeries since longer mixing time slows the rate of the production and also increase the energy cost of mixing. It is impossible to remove salt completely only to have benefits regarding mixing time. Therefore it would be better if the producer do not use high salt content; instead lower salt content would be beneficial. The other option is that to delay salt addition until the dough has reached the clean-up stage.(Miller \& Hoseney, 2008).

\section{The effects of salt on the baking properties of bread}

Salt has multiple effects on the baking properties of bread. As already said, salt increases dough development time, and its resistance to extension and extensibility. Salt also affects bread properties. He et al. (1992) studied the effect of different salt on baking properties of flours that varied widely in baking quality. Sodium sulphate other than sodium chloride improves loaf volume and crumb grain of the bread made from the poor quality flour but made the bread from good quality flour too elastic for good bread-making. Again, salt is not the only factor that can effect bread properties but also the quality of the flour used in the formulation.

Reduced salt content in the formulation does not affect the final bread quality significantly, for example, loaf volume, bake-loss or moisture loss. In addition, omission of salt results in uneven crumb structure and high crumb hardness on 
Helen: The effects of salt on bread

day 5 post-baking. However, these effects are not present when salt is included in the formulation, even at low levels of addition, i.e. $0.6 \%$ or $0.3 \%$. (Lynch, et al., 2009). This means that using reduced salt level in the formulation as low as $0.3 \%$ will have the same effect as using salt in the level usually required for producing good bread which is $1.2 \%$.

\section{The effects of salt on bread flavour}

Finally, the importance for the inclusion of salt in the formulation is that it enhances bread flavour. The flavour enhancing function of salt is well known. Omitting salt from the formulation results in baked products that are quite tasteless. At the level used, salt does not impart salty taste to the product but rather brings out the other flavours in the systems. It is known to increase sweetness and mask metallic bitter or other off flavour (Miller \& Hoseney, 2008). The effect of salt on the palatability of bread may be the important factor considered by bread manufacturer for not producing bread with reduced salt content. According to Lynch et al. 2009 breads are more strongly affected by age than level of salt addition in the sensorial evaluation. Moreover, principle analysis of a descriptive sensory evaluation showed that breads without salt are set in the area of sensorial space described as "sour/acidic, "sourdough" and yeasty flavour attributes. In addition, a study has found that in white bread a reduction in the sodium content of $25 \%$ could remain largely unnoticed as sodium is gradually decreased at the time of baking over a period of 6 weeks (Girgis, et al., 2003). The reduction is achieved without clearly affecting the consumer's perception of the flavour strength and liking of the bread. These results indicate that this could be a promising strategy for sodium reduction in the bread industry.

\section{CONCLUSION}

In conclcusion, salt has important roles to play in affecting yeast activity, strengthening the dough network and thus gas retention of the dough. No technological difficulties are encountered of breads with reduced salt levels. Reducing salt level from 1.2\% (proportion usually used in baking formulation) does not significantly affect dough rheological properties and bread-making quality. However, omission of salt entirely leads to significant reduction in dough, bread quality, and its palatability. 


\section{Recommendations}

The production of bread containing lower salt levels is then technologically feasible, but the taste of the bread needs to be improved. Most of the studies contemplate on the dough and bread properties and few studies focus on the molecular level of gluten properties. Gluten protein molecular structure is indeed affected by salt which may result in different dough and bread properties. Therefore, a study is needed to investigate the effect of different salts on the molecular structure of gluten protein and correlate them with dough rheological and baking properties.

\section{REFERENCES}

Breslin, P. A. S., \& Beauchamp, G. K. (1997). Salt enhances flavour by suppressing bitterness. Nature, 387(6633), 563.

Dotsch, M., Busch, J., Batenburg, M., Liem, G., Tareilus, E., Mueller, R., et al. (2009). Strategies to Reduce Sodium Consumption: A Food Industry Perspective. Critical Reviews in Food Science and Nutrition, 49(10), $841-851$.

Elliott, P., Stamler, J., Nichols, R., Dyer, A. R., Stamler, R., Kesteloot, H., et al. (1996). Intersalt revisited: Further analyses of 24 hour sodium excretion and blood pressure within and across populations.
British Medical Journal, 312(7041), 1249-1253.

Fu, B. X., Sapirstein, H. D., \& Bushuk, W. (1996). Salt-Induced Disaggregation/Solubilization of Gliadin and Glutenin Proteins in Water. Journal of Cereal Science, 24(3), 241-246.

Girgis, S., Neal, B., Prescott, J., Prendergast, J., Dumbrell, S., Turner, C., et al. (2003). A onequarter reduction in the salt content of bread can be made without detection. European Journal of Clinical Nutrition, 57(4), 616-620.

He, H., Roach, R. R., \& Hoseney, R. C. (1992). Effect of nonchoatropic salts on flour bread-making properties. Cereal Chem., 69(4), 366-371.

James, W. P., Ralph, A., \& SanchezCastillo, C. P. (1987). The dominance of salt in manufactured food in the sodium intake of affluent societies. The Lancet, 329(8530), 426-429.

Kinsella, J. E., \& Hale, M. L. (1984). Hydrophobic associations and gluten consistency: Effects of specific anions. Journal of Agricultural and Food Chemistry, 32(5), 1054-1056.

Larsson, H. (2002). Effect of $\mathrm{pH}$ and sodium chloride on wheat flour dough properties: Ultracentrifugation and rheological measurements. Cereal Chemistry, 79(4), 544545. 
Law, M. R., Frost, C. D., \& Wald, N. J. (1991). By how much does dietary salt reduction lower blood pressure? III--Analysis of data from trials of salt reduction. BMJ, 302(6780), 819-824.

Lynch, E. J., Dal Bello, F., Sheehan, E. M., Cashman, K. D., \& Arendt, E. K. (2009). Fundamental studies on the reduction of salt on dough and bread characteristics. Food Research International, 42(7), 885-891.

MacMahon, S., Peto, R., Collins, R., Godwin, J., Cutler, J., Sorlie, P., et al. (1990). Blood pressure, stroke, and coronary heart disease : Part 1, prolonged differences in blood pressure: prospective observational studies corrected for the regression dilution bias. The Lancet, 335(8692), 765-774.

Miller, R. A., \& Hoseney, R. C. (2008). Role of salt in baking. Cereal Foods World, 53(1), 4-6.

Preston, K. R. (1989). Effects of neutral salts of the lyotropic series on the physical dough properties of a Canadian red spring wheat flour. Cereal Chem., 66, 144148.

Salovaara, H. (1982). Effect of partial sodium chloride replacement by other salts on wheat dough rheology and breadmaking. Cereal Chem., 59(5), 422-426.

Tuomilehto, J., Jousilahti, P., Rastenyte, D., Moltchanov, V., Tanskanen, A., Pietinen, P., et al. (2001). Urinary sodium excretion and cardiovascular mortality in Finland: a prospective study. The Lancet, 357(9259), 848851.

Webster, J., Dunford, E., Huxley, R., Li, N., Nowson, C. A., \& Neal, B. (2009). The development of a national salt reduction strategy for Australia. Asia Pacific journal of clinical nutrition, 18(3), 303-309.

WHO (2007). Reducing salt intakes in the population : report of a WHO forum and technical meeting, 5-7 October 2006, Paris, France. Retrieved from http://www.who.int/dietphysical activity/reducingsaltintake_EN.p $\underline{\mathrm{df}}$

Wu, J., Beta, J., \& Clarke, H. (2006). Effects of salt and alkaline reagents on dynamic rhelogical properties of raw oriental noodles Cereal Chem., 83, 211-217. 